\title{
The Geometric Approach for Computing the Joint Spectral Radius
}

\author{
Vladimir Protasov
}

\begin{abstract}
In this paper we describe the geometric approach for computing the joint spectral radius of a finite family of linear operators acting in finite-dimensional Eucledian space. The main idea is to use the invariant sets of of these operators. It is shown that any irreducible family of operators possesses a centrally-symmetric invariant compact set, not necessarily unique. The Minkowski norm generated by the convex hull of an invariant set (invariant body) possesses special extremal properties that can be put to good use in exploring the joint spectral radius. In particular, approximation of the invariant bodies by polytopes gives an algorithm for computing the joint spectral radius with a prescribed relative deviation $\varepsilon$. This algorithm is polynomial with respect to $\frac{1}{\varepsilon}$ if the dimension is fixed.

Another direction of our research is the asymptotic behavior of the orbit of an arbitrary point under the action of all products of given operators. We observe some relations between the constants of the asymptotic estimations and the sizes of the invariant bodies.

In the last section we give a short overview on the extension of geometric approach to the $L_{p}$-spectral radius.
\end{abstract}

\section{INTRODUCTION}

The notion of the joint spectral radius (JSR) of linear operators appeared more than 40 years ago is intensively studied due to many applications in matrix theory, spectral theory, curve and surface design, ergodic theory, etc. For the sake of simplicity we restrict ourselves to the case of two operators $A_{0}, A_{1}$, although all the results are extended for an arbitrary finite number of operators without any change. The notion of JSR can roughly by explained as follows: for a given point $x \in \mathbb{R}^{d}$ it is required to estimate the asymptotic behavior of the value

$$
\max _{d_{1}, \ldots, d_{m}}\left\|A_{d_{1}} \cdots A_{d_{m}}(x)\right\|, \quad m \in \mathbb{N},
$$

where $d_{j}=0$ or 1 for every $j$. In other words, we are interested in how large can the image of the point $x$ under the action of a composition of $m$ operators $A_{0}$ or $A_{1}$ be. It turns out that under some general assumptions on these operators the value (1) have the same exponent of growth for all points $x \neq 0$. It has a polynomial asymptotic growth $\lambda^{m}$. The exponent of growth $\lambda$ is the JSR of operators $A_{0}, A_{1}$.

Definition 1: Let $A_{0}, A_{1}$ be a pair of linear operators acting in $d$-dimensional Euclidean space $\mathbb{R}^{d}$. The following value

$$
\hat{\rho}\left(A_{0}, A_{1}\right)=\lim _{m \rightarrow \infty} \max _{d_{1}, \ldots, d_{m} \in\{0,1\}}\left\|A_{d_{1}} \cdots A_{d_{m}}\right\|^{1 / m}
$$

V.Protasov, Deptartment of Mechanics and Mathematics, Moscow State University, Vorobyovy Gory, Moscow, 119992, Russia, vladimir protassov@yahoo.com

This research was supported by the grant RFBR 05-01-00066 and by the grant 304.2003.1 supporting the leading scientific schools. is the joint spectral radius (JSR) of $A_{0}, A_{1}$.

Denote by $S^{d-1}$ the unit sphere in the space $\mathbb{R}^{d}$ :

$$
S^{d-1}=\left\{x \in \mathbb{R}^{d},\|x\|=1\right\} .
$$

A pair of operators is called irreducible iff they have no common nontrivial real invariant subspace. The problem of computing or estimating of the JSR can actually be considered only for irreducible pairs of operators. Indeed, if the operators $A_{0}, A_{1}$ have a common invariant subspace of dimension $k(0<k<d)$, then their matrices in a suitable basis have the form

$$
A_{i}=\left(\begin{array}{c|c}
B_{i} & D_{i} \\
\hline 0 & C_{i}
\end{array}\right), \quad i=0,1,
$$

where $B_{i}$ are $k \times k$-matrices and $C_{i}$ are $(d-k) \times(d-k)$ matrices. Then it is easily shown that

$$
\hat{\rho}\left(A_{0}, A_{1}\right)=\max \left\{\hat{\rho}\left(B_{0}, B_{1}\right), \hat{\rho}\left(C_{0}, C_{1}\right)\right\}
$$

(see [1], [2]). Therefore, if the operators have common invariant subspaces, then the problem of computing of their JSR is reduced to analogous problems for smaller dimensions. Hence we can restrict ourselves to the case of irreducible operators. Everywhere in the sequel we assume that a pair of operators $A_{0}, A_{1}$ is irreducible.

We begin with the study of the growth of the value (1). In section II we show that in case of irreducible operators this value for any $x \neq 0$ is between $C_{1} \hat{\rho}^{m}$ and $C_{2} \hat{\rho}^{m}$, where $C_{1}, C_{2}$ are some positive constants. Using this result we prove the existence of invariant sets for irreducible operators (section III). Invariant sets have some relation with the notion of affine fractals and self-similar sets and generate the invariant convex bodies and extremal norms (section IV, V). In section VI we give estimations for the constants $C_{1}, C_{2}$ for a given pair of operators, after that we describe an algorithm for computing JSR.

\section{THE ASYMPTOTICS OF THE ORBIT}

Lemma 1: For an irreducible pair of operators $A_{0}, A_{1}$ one has $\hat{\rho}>0$.

Proof: Consider a function $f(x)=$ $\max \left\{\left\|A_{0} x\right\|,\left\|A_{1} x\right\|\right\}$ defined on the unit sphere $S^{d-1}$ and let $a=\min _{x \in S^{d-1}} f(x)$. This minimum is attained, since $f$ is continuous and the sphere is compact. If $a=0$, then the operators have a nontrivial common kernel and hence they are reducible. Whence $a>0$. This yields that for every $m$ one can choose a sequence $d_{1}, \ldots, d_{m}$ such that

$$
\left\|A_{d_{1}} \cdots A_{d_{m}} x\right\| \geq a^{m}\|x\|
$$


hence $\hat{\rho} \geq a$, which completes the proof.

For an arbitrary point $x \in \mathbb{R}^{d}$ and for any $m \geq 1$ let

$$
\mathcal{O}_{m}(x)=\left\{A_{d_{1}} \cdots A_{d_{m}} x, \quad d_{1}, \ldots, d_{m} \in\{0,1\}\right\}
$$

be the orbit of the point $x$ under the action of all possible products of the operators $A_{0}, A_{1}$ consisting of $m$ multipliers. Let

$$
\mathcal{O}(x)=\cup_{m=1}^{\infty} \mathcal{O}_{m}(x)
$$

be the orbit of the point $x$. Also for a set $M \subset \mathbb{R}^{d}$ denote

$$
\|M\|=\max _{y \in M}\|y\| \text {. }
$$

In particular,

$$
\left\|\mathcal{O}_{m}(x)\right\|=\max _{y \in \mathcal{O}_{m}(x)}\|y\|
$$

Proposition 1: For any irreducible pair of operators $A_{0}, A_{1}$ there exist positive constants $C_{1}, C_{2}$, depending only on these operators, such that for every $x \in S^{d-1}, m \geq 1$ one has

$$
C_{1} \hat{\rho}^{m} \leq\left\|\mathcal{O}_{m}(x)\right\| \leq C_{2} \hat{\rho}^{m} .
$$

Proof: Lemma 1 allows us to assume, with possible normalization, that $\hat{\rho}=1$. First let us show that there is a point $x \in S^{d-1}$, for which $\left\|\mathcal{O}_{m}(x)\right\| \leq 2$ for all $m$. If this is not the case, then the union of the sets

$$
U_{m}=\left\{x \in S^{d-1},\left\|\mathcal{O}_{m}(x)\right\|>2\right\}
$$

over all $m \in \mathbb{N}$ covers the unit sphere. Since each of these sets is open, from the compactness of the sphere we conclude that $S^{d-1}=\cup_{m=1}^{N} U_{m}$ for a suitable $N$. Therefore, for every point $x \neq 0$ there exists a product

$$
\Pi=A_{d_{1}} \ldots A_{d_{j}}, \quad j \leq N
$$

such that $\|\Pi(x)\|>2\|x\|$. Now we take an arbitrary point $x_{1} \neq 0$ and consequently find the corresponding products $\Pi_{1}, \Pi_{2}, \ldots$, for which

$$
\left\|\Pi_{k} \cdots \Pi_{1} x_{1}\right\| \geq 2^{k}\left\|x_{1}\right\|, \quad k \in \mathbb{N} .
$$

Since each of these products $\Pi_{j}$ consists of at most $N$ operators, it follows that

$$
\hat{\rho}\left(A_{0}, A_{1}\right) \geq 2^{1 / N},
$$

which contradicts the assumption. Let now $L$ be the set of points $x \in \mathbb{R}^{d}$ that have bounded orbits $\mathcal{O}(x)$. Clearly, $L$ is a linear subspace of $\mathbb{R}^{d}$ invariant with respect to (w.r.t.) the both operators $A_{0}, A_{1}$. Moreover, as we have just shown, $L$ contains at least one nonzero element, i.e., $L$ is nontrivial. Therefore, since the pair $A_{0}, A_{1}$ is irreducible, it follows that $L=\mathbb{R}^{d}$. Thus, the set of operators

$$
\left\{A_{d_{1}} \cdots A_{d_{m}}, d_{j} \in\{0,1\}, m \geq 1\right\}
$$

is bounded at any point $x$, hence, by the Banach-Steinhaus theorem, it is bounded uniformly. This proves the upper bound $\left\|\mathcal{O}_{m}(x)\right\| \leq C_{2}$ for all $x \in S^{d-1}$. To prove the lower bound we apply the same trick. Let us denote

$$
L_{0}=\left\{x \in \mathbb{R}^{d}, \lim _{m \rightarrow \infty}\left\|\mathcal{O}_{m}(x)\right\|=0\right\} .
$$

Again we see that $L_{0}$ is a common invariant subspace for $A_{0}, A_{1}$. Therefore, either it is trivial, or $L_{0}=\mathbb{R}^{d}$. The last case is impossible. Indeed, applying the relation $\lim _{m \rightarrow \infty}\left\|\mathcal{O}_{m}\left(e_{i}\right)\right\|=0$ to the elements of some orthonormal basis $\left\{e_{i}\right\}_{i=1}^{d}$, one can find an integer $N$ such that

$$
\left\|\mathcal{O}_{N}\left(e_{i}\right)\right\|<\frac{1}{2 \sqrt{d}} \text { for all } i=1, \ldots, d .
$$

Therefore $\left\|\mathcal{O}_{N}(x)\right\|<\frac{1}{2}$ for all $x \in S^{d-1}$. Arguing as above we conclude that $\hat{\rho} \leq 2^{-1 / N}$. Thus, $L_{0}$ is trivial. Now assume that there is no positive constant $C_{1}$ such that $\left\|\mathcal{O}_{m}(x)\right\| \geq C_{1}$ for all $m \geq 1, x \in S^{d-1}$. This means that there exists a sequence of points $\left\{x_{k}\right\} \subset S^{d-1}$ and that of numbers $\left\{m_{k}\right\}$ such that $\left\|\mathcal{O}_{m_{k}}\left(x_{k}\right)\right\| \leq \frac{1}{k}$. By the compactness argument it can be assumed that $x_{k}$ converges to some point $x \in S^{d-1}$. We have

$$
\begin{aligned}
& \left\|\mathcal{O}_{m_{k}}(x)\right\| \leq\left\|\mathcal{O}_{m_{k}}\left(x_{k}\right)\right\|+\left\|\mathcal{O}_{m_{k}}\left(x-x_{k}\right)\right\| \\
\leq \frac{1}{k}+C_{2}\left\|x-x_{k}\right\| &
\end{aligned}
$$

Therefore

$$
\left\|\mathcal{O}_{m_{k}}(x)\right\| \rightarrow 0 \quad \text { as } k \rightarrow \infty .
$$

It remains to note that for all $m \geq m_{k}$ we have

$$
\left\|\mathcal{O}_{m}(x)\right\| \leq C_{2}\left\|\mathcal{O}_{m_{k}}(x)\right\|
$$

hence $\left\|\mathcal{O}_{m}(x)\right\| \rightarrow 0$ as $m \rightarrow \infty$, which contradicts the triviality of $L_{0}$. Therefore the proposition follows.

Corollary 1: If a pair of operators $A_{0}, A_{1}$ is irreducible, then

$$
C_{1} \hat{\rho}^{m} \leq \max _{d_{1}, \ldots, d_{m} \in\{0,1\}}\left\|A_{d_{1}} \cdots A_{d_{m}}\right\| \leq C_{2} \hat{\rho}^{m}
$$

for every $m \geq 1$.

\section{INVARIANT SETS}

Our next aim will be to construct invariant sets of linear operators. A compact set $K \subset \mathbb{R}^{d}$ is invariant with respect to a pair of linear operators $A_{0}, A_{1}$ if

$$
A_{0} K \cup A_{1} K=K \text {. }
$$

If $K \neq\{0\}$, then $K$ is called nontrivial.

Proposition 2: An irreducible pair of operators $A_{0}, A_{1}$ possesses a nontrivial invariant set if and only if $\hat{\rho}=1$.

Proof: Necessity. Assume the operators $A_{0}, A_{1}$ possess a nontrivial invariant set $K$. Applying Proposition 1 to an arbitrary point $x \in K \backslash\{0\}$ we get

$$
\left\|\mathcal{O}_{m}(x)\right\| \geq C_{1}\|x\| \hat{\rho}^{m}, \quad m \in \mathbb{N} .
$$

On the other hand, $\mathcal{O}_{m}(x) \subset K$ and hence $\left\|\mathcal{O}_{m}(x)\right\| \leq\|K\|$. This yields $\hat{\rho} \leq 1$. Furthermore, the 
definition of invariant sets implies that for any $x \in K$, there is $y \in K$, for which either $A_{0} y=x$ or $A_{1} y=x$. Consequently, for every $m \geq 1$ there exists $y_{m} \in K$ and a product of our operators

$$
\Pi_{m}=A_{d_{1}} \cdots A_{d_{m}}, \quad d_{j} \in\{0,1\}, j=1, \ldots, m
$$

such that $\Pi_{m} y_{m}=x$. Applying Proposition 1 to the points $y_{m}$ we have

$$
\|x\|=\left\|\Pi_{m}\left(y_{n}\right)\right\| \leq C_{2}\left\|y_{n}\right\| \hat{\rho}^{m} \leq C_{2}\|K\| \hat{\rho}^{m}
$$

for every $m \in \mathbb{N}$. This means $\hat{\rho} \geq 1$ and therefore $\hat{\rho}=1$.

Sufficiency. We assume $\hat{\rho}\left(A_{0}, A_{1}\right)=1$ and prove the existence of a nontrivial invariant set. For an arbitrary point $x \neq 0$ denote by $\mathcal{V}(x)$ the set of points $y \in \mathbb{R}^{d}$ possessing the following property: there exists a sequence $\left\{m_{k}\right\}_{k=1}^{\infty}$ such that the distances from $y$ to the sets $\mathcal{O}_{m_{k}}(x)$ tend to 0 as $k \rightarrow \infty$. Let us show that for any $x$ the set $\mathcal{V}(x)$ is invariant. Proposition 1 guarantees that the set $\mathcal{V}(x)$ is compact and possesses at least one nonzero element. It remains to check that

$$
A_{0} \mathcal{V}(x) \cup A_{1} \mathcal{V}(x)=\mathcal{V}(x) .
$$

Indeed, if $y \in \mathcal{V}(x)$, then $\operatorname{dist}\left(y, \mathcal{O}_{m_{k}}(x)\right) \rightarrow 0$ as $k \rightarrow \infty$, consequently $\operatorname{dist}\left(A_{i} y, A_{i} \mathcal{O}_{m_{k}}(x)\right) \rightarrow 0$ for every $i=0,1$. Since

$$
\operatorname{dist}\left(A_{i} y, \mathcal{O}_{m_{k}+1}(x)\right) \leq \operatorname{dist}\left(A_{i} y, A_{i} \mathcal{O}_{m_{k}}(x)\right),
$$

it follows that

$$
\operatorname{dist}\left(A_{i} y, \mathcal{O}_{m_{k}+1}(x)\right) \rightarrow 0 \quad \text { as } \quad k \rightarrow \infty
$$

and therefore $A_{i} y \in \mathcal{V}(x)$ for each $i=0,1$. Thus,

$$
A_{0} \mathcal{V}(x) \cup A_{1} \mathcal{V}(x) \subset \mathcal{V}(x)
$$

To prove the inverse inclusion we take an arbitrary $y \in \mathcal{V}(x)$ and consider the operator products $\Pi_{m_{k}}$, for which $\Pi_{m_{k}}(x) \rightarrow y$ as $k \rightarrow \infty$. Without loss of generality it can be assumed that infinitely many of these products begin with the operator $A_{0}$. Passing, if necessary, to a subsequence we suppose that all the products $\Pi_{m_{k}}$ begin with $A_{0}$, i.e.,

$$
\Pi_{m_{k}}=A_{0} \Pi_{m_{k}-1}, \quad k \geq 2 .
$$

The sequence of points $\Pi_{m_{k}-1}(x)$ is bounded, therefore it has a subsequence that converges to some point $y_{0}$. Passing to this subsequence we can assume

$$
\Pi_{m_{k}-1}(x) \rightarrow y_{0} \quad \text { as } \quad k \rightarrow \infty .
$$

Whence $y_{0} \in \mathcal{V}(x)$ and $A_{0} y_{0}=y$. Thus, for any point $y \in \mathcal{V}(x)$ there exists either a point $y_{0}$ or $y_{1}$ from the set $\mathcal{V}(x)$ such that $A_{i} y_{i}=y(i=0,1)$. This proves the inverse inclusion

$$
\mathcal{V}(x) \subset A_{0} \mathcal{V}(x) \cup A_{1} \mathcal{V}(x)
$$

Thus, for any $x \neq 0$ the set $\mathcal{V}(x)$ is invariant for the operators $A_{0}, A_{1}$.

Remark 1: The definition of invariant sets coincides with the definition of fractals given by J.E.Hutchinson in [3].
According to the classical results of that work every finite set of contraction affine operators possesses a unique fractal (self-similar set). In contrast to that, in our situation the operators are neither affine nor contraction but linear with the condition $\hat{\rho}=1$. The other distinction is that in our case the invariant sets are not unique. If $K$ is an invariant set, then for any $\lambda>0$ the set $\lambda K$ also is. However, the invariant set may not be unique even up to multiplication to a scalar. Moreover, for contraction affine operators one has the convergence

$$
\tilde{A}^{m} X \rightarrow K, \quad k \rightarrow \infty
$$

in the Hausdorff metric for an arbitrary compact set $X \subset$ $\mathbb{R}^{d}$, where $\tilde{A} X=A_{0} X \cup A_{1} X$ [3]. For linear operators with $\hat{\rho}=1$ and for their invariant set $K$ this is not necessarily the case. This is seen already from the following simplest examples:

Example 1: Both $A_{0}, A_{1}$ are rotations of the plane $\mathbb{R}^{2}$ on rational angles ( $\pi q$, where $q$ is rational). Then both the unit ball and a suitable regular polygon are invariant sets for $A_{0}, A_{0}$.

Example 2: Both $A_{0}, A_{1}$ are orthogonal projectors of the plane $\mathbb{R}^{2}$ on two straight lines $a_{0}, a_{1}$ (these lines are not orthogonal). Then any parallelogram, having its diagonals on these lines and such that its sides form acute angles with the diagonals, is an invariant set for $A_{0}, A_{1}$.

Example 3: Let $A_{0}$ be a rotation of the plane $\mathbb{R}^{2}$ on the angle $\frac{\pi}{2}, A_{1}$ be the reflection with respect to a line $b$ passing trough the origin, $I_{0}, I_{1}$ be two equal segments forming the angles $\frac{\pi}{4}$ with the line $b$ and having their common midpoint at the origin. Since

$$
A_{i} I_{0}=I_{1}, \quad A_{i} I_{1}=I_{0},
$$

for $i=0,1$ it follows that the sequence $\tilde{A}^{m} I_{0}$ does not converge at all.

\section{INVARIANT BODIES AND EXTREMAL NORMS}

We call a set $M \subset \mathbb{R}^{d}$ convex body if it is convex, compact, and possesses a nonempty interior. Everywhere below we consider only convex bodies centrally symmetric with respect to the origin.

Let us consider the following binary operation on the set of (centrally symmetric) convex bodies:

$$
\bar{A} M=\operatorname{Conv}\left(A_{0} M, A_{1} M\right),
$$

where Conv is the convex hull. Since our pair of operators is irreducible, it follows that $\bar{A}$ takes convex bodies to convex bodies. An invariant body is a convex body $M$ such that $\bar{A}=\lambda M$ for some positive $\lambda$.

Theorem 1: a) An irreducible pair of operators always possesses an invariant body.

b) For any invariant body we have $\lambda=\hat{\rho}\left(A_{0}, A_{1}\right)$.

Proof: With possible normalization it can be assumed that $\hat{\rho}=1$. By Proposition 2 the operators $A_{0}, A_{1}$ possess 
an invariant set $K$. It follows that $M=\operatorname{Conv}(K,-K)$ is their invariant body with $\lambda=1$. This set has a nonempty interior, otherwise it is contained in some subspace $L \subset \mathbb{R}^{d}$ of smaller dimension, which is a common invariant subspace for $A_{0}, A_{1}$. This proves part (a). Now let us establish (b). If $M$ is an invariant body of our pair of operators, then in the Minkowski norm $\|\cdot\|_{M}$ defined by this body one has

$$
\left\|A_{i}\right\|_{M} \leq \lambda, \quad i=0,1
$$

therefore $\hat{\rho} \leq \lambda$, and so $\lambda \geq 1$. On the other hand, by Proposition 1 , for any $k \geq 1$ we have

$$
C_{2} \cdot\|M\| \geq\left\|\bar{A}^{k} M\right\|=\lambda^{k} \cdot\|M\|,
$$

and hence $\lambda \leq 1$.

Example 4: If the operators coincide, i.e., $A_{0}=A_{1}=A$, then they possess an invariant body if and only if the modules of all eigenvalues of $A$ are equal and its Jordan form has only diagonal elements [2]. Observe that Theorem 1 is not extended to this case, whenever $d \geq 3$, since A possesses an invariant subspace of dimension 1

Remark 2: In some sense an invariant body $M$ gives the smallest invariant norm in $\mathbb{R}^{d}$ for the operators $A_{0}, A_{1}$. In general an invariant body of a given pair of operators is not unique, even up to normalization. Examples 1, 2, 4 treat very simple cases of invariant bodies. For general pairs of operators the structure of invariant bodies must be complicated, their boundaries possess fractal-like properties.

\section{THE STRUCTURE OF INVARIANT BODIES}

Proposition 2 suggests a method of approximation of invariant bodies. It was shown in [2] that that for an arbitrary point $x \neq 0$ the set

$$
\cup_{k=m}^{+\infty} \mathcal{O}_{k}(x)
$$

converges to $\mathcal{V}(x)$ in the Hausdorff metric as $m \rightarrow \infty$. This implies, in particular, that for large $m$ the set $\cup_{k=m}^{2 m} \mathcal{O}_{k}(x)$ gives a good approximation for the invariant set $\mathcal{V}(x)$. Consequently, taking a convex hull of this set with its symmetric w.r.t. the origin, we get an approximation for the invariant body. This approach was put to good use in the works of I.Sheipak, where concrete examples and pictures were provided (see [4] and references therein).

To clarify the structure of invariant sets and of invariant bodies one can use the notion of returning points. Assume again $\hat{\rho}=1$. A point $x \neq 0$ is called returning if $x \in \mathcal{V}(x)$. In other words, suitable compositions of the operators $A_{0}, A_{1}$ take the point $x$ close to itself. The following result was established in [2].

Proposition 3: An irreducible pair of operators normalized by the condition $\hat{\rho}=1$ has at least one returning point. For any set of returning points $\mathcal{W}$ the set $\cup_{x \in \mathcal{W}} \mathcal{O}(x)$ is invariant. Moreover, every invariant set of our operators have this form. In particular, every invariant body is represented as

$$
M=\operatorname{Conv}\{\mathcal{O}(x),-\mathcal{O}(x) \mid x \in \mathcal{W}\}
$$

for a suitable set of returning points $\mathcal{W}$.

As a corollary of Proposition 3 we have

Theorem 2: For any pair of operators $A_{0}, A_{1}$ one has

$$
\hat{\rho}^{-m} \max _{d_{1}, \ldots, d_{k}, k \leq m} \rho\left(A_{d_{1}} \cdots A_{d_{k}}\right) \rightarrow 1, \quad m \rightarrow \infty,
$$

where $\rho(A)$ is the (usual) spectral radius of the operator $A$.

Proof: It suffices to establish this theorem for irreducible pairs of operators (the general case is easily reduced to this one by using decomposition (2)). With possible normalization it can be assumed that $\hat{\rho}\left(A_{0}, A_{1}\right)=1$. For any product $\Pi_{k}=A_{d_{1}} \ldots A_{d_{k}}$ one has $\rho\left(\Pi_{k}\right) \leq 1$ (see, for instance, [1]). Let $x \neq 0$ be a returning point. There is a sequence of operator products $\left\{\Pi_{k}\right\}_{k \in \mathbb{N}}$ such that

$$
\Pi_{k}(x) \rightarrow x, \quad k \rightarrow \infty .
$$

On the other hand, the norms of all the operators $\left\|\Pi_{k}\right\|$ are uniformly bounded by the constant $C_{2}$ (Corollary 1). It now follows that each operator $\Pi_{k}$ possesses an eigenvector $x_{k}$ such that $x_{k} \rightarrow x$ and the corresponding eigenvalue $\lambda_{k}$ tends to 1 as $k \rightarrow \infty$. Therefore $\rho\left(\Pi_{k}\right) \rightarrow 1$, which concludes the proof.

Remark 3: Theorem 2 slightly sharpens the well-known relation

$$
\max _{d_{1}, \ldots, d_{m}}\left[\rho\left(A_{d_{1}} \cdots A_{d_{m}}\right]^{1 / m} \rightarrow \hat{\rho}, \quad m \rightarrow \infty .\right.
$$

(see [1] for the proof). This relation is used in most of practical algorithms of computing JSR. Therefore, it is important to estimate the rate of convergence. Theorem 2 implies the following asymptotics:

$$
\mid \hat{\rho}-\max _{d_{1}, \ldots, d_{k}, k \leq m}\left[\rho\left(A_{d_{1}} \cdots A_{d_{k}}\right]^{1 / k} \mid=o\left(\frac{1}{m}\right), \quad m \rightarrow \infty\right.
$$

\section{ESTIMATIONS FOR $C_{1}$ AND $C_{2}$}

Let $\partial X$ denote the boundary of a set $X$. For any convex body $M$ set

$$
l(M)=\frac{\max _{x \in \partial M}\|x\|}{\min _{x \in \partial M}\|x\|} .
$$

For an irreducible pair of operators $A_{0}, A_{1}$ its invariant body $M$ is not unique, nevertheless the value $l(M)$ is bounded uniformly for all invariant bodies. In fact, there is an effective constant $H$ depending only on operators $A_{0}, A_{1}$ such that for any invariant body we have $l(M) \leq H$. In some sense, the constant $H$ measures the "irreducibility" of this pair of operators. In [2] it was shown how to compute $H$ effectively in terms of real eigenspaces of the operators $A_{0}, A_{1}$. This constant is expressed in terms of angles between real eigenspaces of these operators.

Theorem 3: For an irreducible pair of operators $A_{0}, A_{1}$ one has

$$
\hat{\rho}^{m} \leq \max _{d_{1}, \ldots, d_{m}}\left\|A_{d_{1}} \cdots A_{d_{m}}\right\| \leq H \hat{\rho}^{m}, \quad m \in \mathbb{N} .
$$

Proof: The left-hand side inequality is well known. To prove the second inequality we take an arbitrary point 
$x \in \mathbb{R}^{d} \backslash\{0\}$ and an invariant body $M$ of the operators $A_{0}, A_{1}$. With possible normalization it can be assumed that $x \in \partial M$. Since for any $\Pi_{m}=A_{d_{1}} \cdots A_{d_{m}}$ we have

$$
\Pi_{m} x \in \hat{\rho}^{m} M
$$

it follows that $\left\|\Pi_{m} x\right\| \leq l(M) \hat{\rho}^{m}$.

Clearly, $H$ provides also an upper bound for the constant $C_{2}$ in inequality (3). In [2] a lower bound for the constant $C_{1}$ was given.

\section{THE ALGORITHM OF COMPUTING JSR}

One of the most intriguing problem concerning the JSR is the complexity of its computation or estimation. Most of algorithms used in practical problems compute JSR using relation (4), by exhaustion of all matrix products of a given length $m$. Several modifications of this algorithm were elaborated in [5], [6], [7]. However, all the estimations for the rate of convergence of (4) available by now give exponential upper bounds of its complexity. In fact, the problem of computing JSR is NP-hard, and there is no algorithm that would be polynomial with respect to both the dimension $d$ and the relative error $\varepsilon$ [8], [9].

Theorems 1 and 3 make it possible to construct the geometric algorithm of computing JSR. The idea of the algorithm is simple and consists of iterative approximation of the invariant body by polytopes. If the dimension $d$ is fixed, then the algorithm is polynomial w.r.t. the $\frac{1}{\varepsilon}$, where $\varepsilon$ is a given accuracy.

The Algorithm. Let us have an irreducible pair of operators $A_{0}, A_{1}$ and an accuracy $\varepsilon>0$. It is required to find a number $\hat{\rho}^{*}$ such that

$$
\frac{\left|\hat{\rho}^{*}-\hat{\rho}\right|}{\hat{\rho}}<\varepsilon .
$$

Zero step. Take a cross-polytope

$$
T_{0}=\left\{\left(x_{1}, \ldots, x_{d}\right) \in \mathbb{R}^{d}, \quad \sum\left|x_{i}\right| \leq 1\right\} .
$$

We apply $A_{0}$ and $A_{1}$ to this polytope and take the convex hull of the images $A_{0} T_{0}, A_{1} T_{0}$. We obtain the polytope $\bar{A} T_{0}$. If the number of its vertices does not exceed $q(\varepsilon)=C_{d} \varepsilon^{\frac{1-d}{2}}$ ( $C_{d}$ is an effective constant depending only on $d$ ), then we set $T_{1}=\bar{A} T_{0}$. If it does, then find a polytope $T$ such that it has at most $q(\varepsilon)$ vertices and

$$
(1-\varepsilon) \bar{A} T_{0} \subset T \subset \bar{A} T_{0},
$$

and put $T_{1}=T$.

$m$ th step. We have a polytope $T_{m}$ with at most $q(\varepsilon)=$ $C_{d} \varepsilon^{\frac{1-d}{2}}$ vertices. Consider the polytope $\bar{A} T_{m}$. If the number of its vertices does not exceed $q(\varepsilon)$, then $T_{m+1}=\bar{A} T_{m}$, otherwise construct a polytope $T_{m+1}$ that has at most $q(\varepsilon)$ vertices and

$$
(1-\varepsilon) \bar{A} T_{m} \subset T_{m+1} \subset \bar{A} T_{m} .
$$

After $N=\left[\frac{3 \sqrt{d} \ln H}{\varepsilon}\right]$ steps the algorithm terminates. The value $\hat{\rho}^{*}=\left(v_{N+1}\right)^{1 /(N+1)}$ gives the desirable approximation of JSR, where $v_{k}$ is the biggest distance from the origin to the vertices of the polytope $T_{k}$.

Each step requires us taking of a convex hull of two polytopes having at most $q(\varepsilon)$ vertices and the approximation of one polytope with $2 q(\varepsilon)$ vertices by a different one with $q(\varepsilon)$ vertices with accuracy $\varepsilon$ in the sense of relation (5). Both operations are known to be polynomial w.r.t. $\frac{1}{\varepsilon}$ (the dimension $d$ is fixed).

The case $d=2$ is easily implemented. In this case $C_{d} \leq 11$ and so $q(\varepsilon)=11 / \sqrt{\varepsilon}$. The algorithm of computing $\hat{\rho}^{*}$ takes at most $C_{0} \varepsilon^{-3 / 2}$ arithmetic operations, where $C_{0}$ is an effective constant depending on operators $A_{0}, A_{1}$. Theoretical explanations and the estimations of the complexity can be found in [2], some examples of implementation - in [4].

\section{VIII. $p$-RADIUS}

Geometric approach using invariant bodies is also applicable in exploring the following natural generalization of the joint spectral radius:

Definition 2: For a given $p \in[1,+\infty)$ the $L^{p}$-spectral radius ( $p$-radius) of linear operators $A_{0}, A_{1}$ is the value

$$
\rho_{p}\left(A_{0}, A_{1}\right)=\lim _{m \rightarrow \infty}\left(2^{-m} \sum_{d_{1}, \ldots, d_{m}}\left\|A_{d_{1}} \cdots A_{d_{m}}\right\|^{p}\right)^{1 / p m} .
$$

The $p$-radius was first introduced for $p=1$ and then generalized for all $p \geq 1$. It is applied in wavelets theory and approximation theory to characterize the regularity of wavelets and scaling functions in the space $L^{p}$ (see [12], [13] for details and further references). Actually, the joint spectral radius $\hat{\rho}$ can be considered as the $p$-radius for $p=\infty$, in particular $\lim _{p \rightarrow \infty} \rho_{p}=\hat{\rho}$. Besides, for any $p$ we obviously have

$$
\rho_{p} \leq \hat{\rho} \leq 2^{1 / p} \rho_{p}
$$

Let us remark that the $p$-radius is not expressed by a limit passage from the regular spectral radius as we have for the JSR (relation (4)). That is why there was actually no algorithms for computing of $p$-radius except for by definition.

In [13] and [14] the geometric approach and the conception of invariant bodies was extended to the $p$-radius for all $p \geq 1$. Instead of the operation Conv the so-called Firey addition $\stackrel{p}{\oplus}$ (sometimes it is called $L^{p}$-summation) was used. If $M_{1}, M_{2}, M_{3} \subset \mathbb{R}^{d}$ are convex bodies (symmetric w.r.t. the origin), then by definition $M_{3}=M_{1} \stackrel{p}{\oplus} M_{2}$ iff $\varphi_{M_{3}}=\left\|\left(\varphi_{M_{1}}, \varphi_{M_{2}}\right)\right\|_{p}$, where $\varphi_{M}(x)=\sup _{y \in M}\langle x, y\rangle$ is the function of support of a convex body $M$ and $\|\cdot\|_{p}$ is the $L^{p}$-norm. Clearly, for $p=\infty$ the Firey addition coincides with the taking of convex hull, for $p=1$ that coincides with the Minkowski summation. In some sense, the Firey addition is the only reasonable generalization of the taking of convex hull and of the Minkowski summation [12]. 
Theorem 4: For an irreducible pair of operators $A_{0}, A_{1}$ acting in $\mathbb{R}^{d}$ and for any $p \geq 1$ there exists a convex body $M_{p}$ and $\lambda>0$ such that

$$
A_{0} M_{p} \stackrel{p}{\oplus} A_{1} M_{p}=\lambda M_{p} .
$$

Moreover, for every such convex body we have $\lambda=2^{1 / p} \rho_{p}$.

Thus, an irreducible pair of linear operators for any $p$ possesses an invariant body (not necessarily unique) corresponding to their $p$-radius. As a corollary we get the following generalization of Proposition 1:

Theorem 5: Suppose $A_{0}, A_{1}$ by an irreducible pair of operators and $p \geq 1$; then there are positive constants $C_{1}, C_{2}$ depending only on $A_{0}, A_{1}$ and on $p$ such that for any point $x \in S^{d-1}$ and for all $m$ we have

$C_{1}\left(\rho_{p}\right)^{m p} \leq 2^{-m} \sum_{d_{1}, \ldots, d_{m}}\left\|A_{d_{1}} \cdots A_{d_{m}} x\right\|^{p} \leq C_{2}\left(\rho_{p}\right)^{m p}$.

Theorems 4 and 5 were proved in [13], also some estimations for the constants $C_{1}, C_{2}$ were established. Based on these theorems a geometric algorithm for computing the $p$-radius was derived. The algorithm consists of iterative approximation of the invariant body by $p$-zonotopes (Firey sums of finitely many segments). This is also polynomial w.r.t. the $1 / \varepsilon$, the number of operations does not exceed $C(d) \varepsilon^{2-2 d}$.

\section{REFERENCES}

[1] M. A. Berger, Y. Wang, Bounded semi-groups of matrices, Linear Alg. Appl., 166 (1992), pp. 21-27.

[2] V. Yu. Protasov, The joint spectral radius and invariant sets of linear operators, Fundamentalnaya i prikladnaya matematika, 2 (1996), No 1, pp. 205-231.

[3] J.E. Hutchinson, Fractals and self-similarity, Indiana Univ. Math. J., 30 (1981), No 5, pp. 713-747.

[4] I.Sheipak, Nontrivial fractals in the plane and linear operators with joint spectral radius equal to 1, Math. Notes 63 (1998), No.5, pp. 701705 .

[5] G. Gripenberg, Computing the joint spectral radius, Lin. Alg. Appl., 234 (1996), pp. 43-60.

[6] I. Daubechies, J.Lagarias, Two-scale difference equations. II. Local regularity, infinite products of matrices and fractals, SIAM. J. Math. Anal. 23 (1992), 1031-1079.

[7] D.Collela, C.Heil, Characterization of scaling functions. I. Continuous solutions, SIAM J. Matrix Anal. Appl., 15 (1994), 496-518.

[8] V. Blondel, J. M. Tsitsiklis, Approximating the spectral radius of sets of matrices in the max-algebra is NP-hard, IEEE Trans. Autom. Control., 45 (2000), No 9, pp. 1762-1765.

[9] V. Blondel, J. M. Tsitsiklis, The boundedness of all products of a pair of matrices is undecidable, Syst. Control Lett., 41 (2000), No 2, pp. $135-140$.

[10] K.S. Lau, J. Wang, Characterization of $L_{p}$-solutions for two-scale dilation equations, SIAM J. Math. Anal. 26 (1995), pp. 1018-1046.

[11] Y.Wang, Two-scale dilation equations and the mean spectral radius. Random Comput.Dynamic, 4 (1996),No 1, pp.49-72

[12] V. Yu. Protasov, Possible generalizations of the convex addition, Bulletin of Moscow University. Mathematics and Mechanics., 4 (1999), pp. 13-19.

[13] V. Yu. Protasov, The generalized spectral radius. The geometric approach, Izvestiya Mathematics. 61 (1997), No 5, pp. 995-1030.

[14] V. Yu. Protasov, A generalization of the joint spectral radius: the geometrical approach, Facta Univ. Ser. Math. Inform. No. 13 (1998), pp. 19-23. 